\title{
Gaussian Process Regression Applied to VRLA Battery Voltage Prediction in Photovoltaic Off-Grid Systems
}

\author{
Iván Sanz ${ }^{1}$, Carlos Bernal ${ }^{1}$, Antonio Bonoํ, Milutin Pajovic ${ }^{3}$, Gabriel Martínez ${ }^{1}$ \\ ${ }^{1}$ Grupo de Electrónica de Potencia y Microelectrónica (GEPM); \\ ${ }^{2}$ Human OpenWare Research Lab (HOWLab) \\ Instituto de Investigación en Ingeniería de Aragón (I3A) \\ Universidad de Zaragoza, Mariano Esquillor s/n, 50018, Zaragoza, Spain. \\ Tel. +34-976762707, e-mail: isgorra@unizar.es \\ ${ }^{3}$ Mitsubishi Electric Research Laboratory (MERL), Boston, MA, USA.
}

\begin{abstract}
This study addresses the use of GPR techniques for VRLA battery voltage prediction purposes in PV offgrid systems. The goal is to know whether the system is able to endure a predictable power consumption pattern without running out of energy. Two approaches are considered: sample based prediction and pattern-based forecasting.
\end{abstract}

\section{Introduction}

Gaussian Process Regression algorithms are being applied to the battery field. Specifically, to the problem of future battery voltage prediction, as the final voltage after a given current pattern, among other applications as State-of-Charge or End-of-Life estimation $[1,2]$. In these studies, the algorithms have been tested with data from laboratory tests, synthetic data form a previous model and also with data from a NASA database, and are focused on Lithium-Ion technologies.

This study is going to consider the same kernel functions that have been proposed in these previous work, to extend the application of the GPR techniques and their capabilities to model and predict Valve Regulated Lead Acid (VRLA) battery voltage. The algorithm is going to be tested with a photovoltaic off-grid (PV) dataset, which contains 10 years of past current, voltage and temperature waveforms sampled on hour basis in different photovoltaic off-grid systems. Those systems are designed to supply communication sites along Ebro basin. The final goal is to know whether the system will endure a whole night without energy disruption.

Some different approaches are going to be followed. First, the algorithm is used for voltage waveform prediction, using a similar recursive GPR algorithm than in other applications. Then, the problem of future current samples is dealt with. To finish, the algorithm has been tested trying to predict some key features of the waveforms, instead of the whole time series.

\section{Voltage Waveform Prediction}

Inspired by the previous work, a first approach based on voltage waveform prediction is considered. The start point is a core algorithm, capable of predicting voltage sample at $t+1$ from previous windowed waveforms of current, voltage and temperature, along with future current. Then, the algorithm is used recursively to predict the voltage at $t+n$, where $n$ is the desired prediction horizon. In this paper, a prediction horizon of 24 hours is being considered.

Once the core algorithm has been trained and matches the application, the problem of knowing the future current needs to be dealt with. The power consumption is constant and known in this application, but the power generation is not (it depends on the sun). The current generation patterns in the database have been studied and pre-processed using a Self-Organizing Map (SOM) and the k-means algorithm. These are classic clustering techniques [3] and through them, a generation pattern classification has been established (Fig. 1), consisted of five pattern types:

- Low irradiation days

- Winter days with float stage

- Winter days without float stage

- Spring days

- Summer days

A GPR processing bank formed by five parallel GPR estimators has been developed, each current profile is used as future current input. Each of the algorithms predicts the future evolution of the battery voltage. These predicted waveforms can be combined afterwards, obtaining the final predicted waveform (Fig. 2). Error metrics are collected in Table $1 \mathrm{col} 1$ (48V battery nominal voltage). 
When trying to predict the whole waveform, error metrics are distorted because of the uncertainty regarding the charge process. However, the discharge process has a much more controlled waveform. This can be appreciated in Fig. 2, where voltage estimations during discharge processes are more accurate than during the charge process. The error metrics obtained after computing only the discharge waveform are collected in Table $1 \mathrm{col} 2$.

\section{Feature prediction}

The obtained metrics show that the discharge prediction is much more accurate than the charge prediction. As in this application the goal is to know if the system is going to ran out of energy, the discharge process is critical. For this purpose, a different approach is going to be developed. Instead of trying to predict the whole voltage waveform, the new algorithm is going to focus in the value of the voltage at the end of the night (Fig. 2, green values). A GPR algorithm has been off-line trained for this purpose. Its inputs are features of the 7-daywindowed waveforms:

- Daily stored charge

- Start-of-Discharge Voltage

- End-of-Discharge Voltage

- Temperature along charge and discharge processes

- Duration of the charge and discharge processes

With this approach, the error metrics achieved are displayed in Table $1 \mathrm{col} 3$, and only one GPR algorithm is needed, using less computing resources. In this case, the MAPE metric grows, since the range of variation of the discharge voltage is smaller than the range of the whole voltage waveform; but the RMSE remains low.

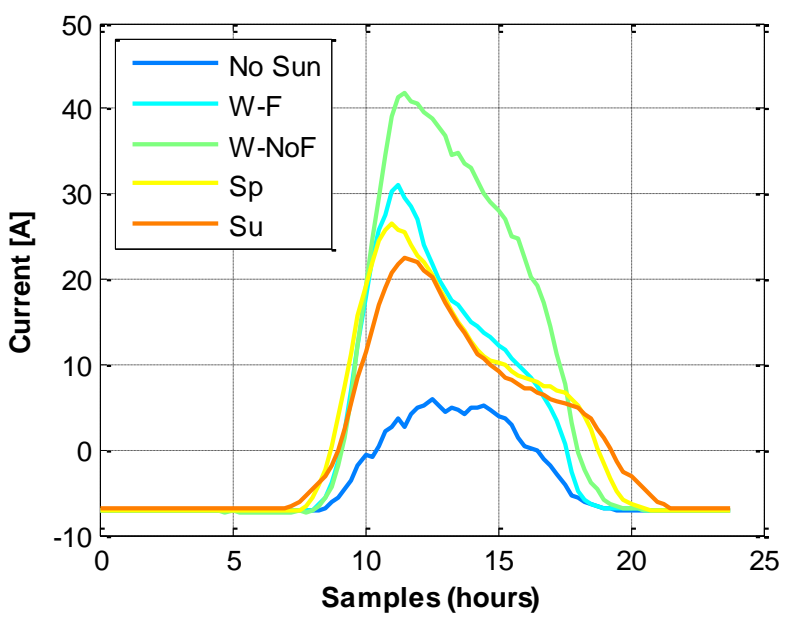

Fig. 1. Day Classification
Table 1. Error metrics for each type of prediction

\begin{tabular}{|l|c|c|c|}
\hline $\begin{array}{l}\text { Error } \\
\text { metric }\end{array}$ & $\begin{array}{l}\text { Waveform } \\
\text { prediction }\end{array}$ & $\begin{array}{l}\text { Discharge } \\
\text { waveform }\end{array}$ & $\begin{array}{l}\text { Feature } \\
\text { prediction }\end{array}$ \\
\hline MAPE & $7.53 \%$ & $1.61 \%$ & $4.23 \%$ \\
\hline RMSE & $1.37 \mathrm{~V}$ & $194 \mathrm{mV}$ & $187 \mathrm{mV}$ \\
\hline
\end{tabular}

\section{Conclusions}

GPR techniques have proven to be a useful and accurate tool to model and predict battery voltage in $\mathrm{PV}$ installations. The traditional waveform prediction approach may not be as useful for this specific applications, due to the uncertainty in the power generation profiles. However, the discharge profiles are adequately predicted, with low RMS error. Instead of waveform prediction, a new and simpler approach is introduced. It is based in feature prediction, and the error metrics are similar to the traditional approach in discharge, while the computing resources required are less demanding.

\section{REFERENCES}

[1] OZCAN, Gozde, PAJOVIC, Milutin, SAHINOGLU, Zafer, WANG, Yebin, ORLIK, Philip V and WADA, Toshihiro. Online State of Charge Estimation for Lithium-Ion Batteries Using Gaussian Process Regression. . 2016. P. 0-5.

[2] PAJOVIC, Milutin, SAHINOGLU, Zafer, WANG, Yebin, ORLIK, Philip V. and WADA, Toshihiro. Online data-driven battery voltage prediction. Proceedings - 2017 IEEE 15th International Conference on Industrial Informatics, INDIN 2017. 2017. P. 827-834. DOI 10.1109/INDIN.2017.8104879.

[3] KOHONEN, Teuvo. Self-Organizing Maps. Berlin, Heidelberg: Springer Berlin Heidelberg, 2001. Springer Series in Information Sciences. ISBN 978-3540-67921-9.

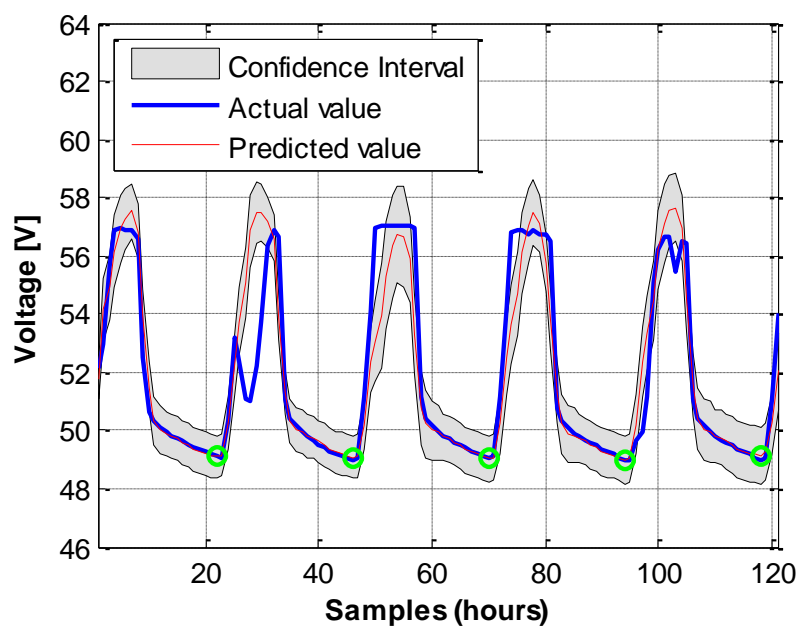

Fig 2. Predicted waveforms

Revista "Jornada de Jóvenes Investigadores del I3A", vol. 6 (Actas de la VII Jornada de Jóvenes Investigadores del I3A - 8 de junio de 2018). ISSN 2341-4790. 